\title{
LOOSENESS AND INDEPENDENCE NUMBER OF TRIANGULATIONS ON CLOSED SURFACES
}

\author{
Atsuhiro Nakamoto, Seiya Negami \\ Graduate School of Environment and Information Science \\ Yokohama National University \\ 79-7 Tokiwadai, Hodogaya-Ku, Yokohama 240-8501, Japan \\ e-mail: nakamoto@ynu.ac.jp, negami@ynu.ac.jp \\ Kyoji OHBA \\ Yonago National College of Technology \\ Yonago, Tottori 683-8502, Japan \\ e-mail: ooba@yonago-k.ac.jp
}

AND

Yusuke SuzukI

Department of Mathematics

Niigata University

8050 Ikarashi 2-no-cho, Nishi-ku, Niigata, 950-2181, Japan

e-mail: y-suzuki@math.sc.niigata-u.ac.jp

\begin{abstract}
The looseness of a triangulation $G$ on a closed surface $F^{2}$, denoted by $\xi(G)$, is defined as the minimum number $k$ such that for any surjection $c: V(G) \rightarrow\{1,2, \ldots, k+3\}$, there is a face uvw of $G$ with $c(u), c(v)$ and $c(w)$ all distinct. We shall bound $\xi(G)$ for triangulations $G$ on closed surfaces by the independence number of $G$ denoted by $\alpha(G)$. In particular, for a triangulation $G$ on the sphere, we have

$$
\xi(G) \leq \frac{11 \alpha(G)-10}{6}
$$

and this bound is sharp. For a triangulation $G$ on a non-spherical surface $F^{2}$, we have

$$
\xi(G) \leq 2 \alpha(G)+l\left(F^{2}\right)-2,
$$

where $l\left(F^{2}\right)=\left\lfloor\left(2-\chi\left(F^{2}\right)\right) / 2\right\rfloor$ with Euler characteristic $\chi\left(F^{2}\right)$.
\end{abstract}


Keywords: triangulations, closed surfaces, looseness, $k$-loosely tight, independence number.

\section{Mathematics Subject Classification: 05C10.}

\section{REFERENCES}

[1] K. Appel, W. Haken and J. Koch, Every planar map is four colorable, Illinois J. Math. 21 (1977) 429-567.

[2] J.L. Arocha, J. Bracho and V. Neumann-Lara, On the minimum size of tight hypergraphs, J. Graph Theory 16 (1992) 319-326. doi:10.1002/jgt.3190160405

[3] J.L. Arocha, J. Bracho and V. Neumann-Lara, Tight and untight triangulations surfaces by complete graphs, J. Combin. Theory Ser. B 63 (1995) 185-199. doi:10.1006/jctb.1995.1015

[4] J. Czap, S. Jendrol', F. Kardoš and J. Miškuf, Looseness of plane graphs, Graphs Combin. 27 (2011) 73-85.

doi:10.1007/s00373-010-0961-6

[5] S. Negami and T. Midorikawa, Loosely-tightness of triangulations of closed surfaces, Sci. Rep. Yokohama Nat. Univ., Sec. I 43 (1996) 25-41.

[6] S. Negami, Looseness ranges of traingulations on closed surfaces, Discrete Math. 303 (2005) 167-174.

doi:10.1016/j.disc.2005.01.010

[7] G. Ringel, Map Color Theorem (Springer-Verlag, Berlin Heidelberg, 1974). doi:10.1007/978-3-642-65759-7

[8] T. Tanuma, One-loosely tight triangulations on closed surfaces, Yokohama Math. J. 47 (1999) 203-211.

Received 13 April 2015

Revised 8 September 2015

Accepted 8 September 2015 